Article

\title{
Electromagnetic Waves in Annular Regions
}

\author{
Daniele Funaro \\ Dipartimento di Scienze Chimiche e Geologiche, Università di Modena e Reggio Emilia, Via Campi 103, \\ 41125 Modena, Italy; daniele.funaro@unimore.it
}

Received: 4 February 2020; Accepted: 1 March 2020; Published: 5 March 2020

\begin{abstract}
In suitable bounded regions immersed in vacuum, time periodic wave solutions solving a full set of electrodynamics equations can be explicitly computed. Analytical expressions are available in special cases, whereas numerical simulations are necessary in more complex situations. The attention here is given to selected three-dimensional geometries, which are topologically equivalent to a toroid, where the behavior of the waves is similar to that of fluid-dynamics vortex rings. The results show that the shape of the sections of these rings depends on the behavior of the eigenvalues of a certain elliptic differential operator. Time-periodic solutions are obtained when at least two of such eigenvalues attain the same value. The solutions obtained are discussed in view of possible applications in electromagnetic whispering galleries or plasma physics.
\end{abstract}

Keywords: electromagnetism; periodic waves; toroid; vortex rings; confined solutions

\section{Introduction}

We collect here some mathematical results concerned with the development of solitary electromagnetic waves confined in bounded regions of space having a toroid shape. We start by pointing out that exact analytic solutions are available in the case of an unbounded cylinder (which is, hypothetically, a toroid closed at infinity), whereas for bounded rings some numerical assistance is necessary. The structures here considered display a strong analogy with fluid dynamics vortex rings (many classical texts are available as possible references). Indeed, vortex tori are amazing substructures. The flow lies inside a well determined doughnut-shaped domain and follows a rotatory motion around the major circumference. Usually, sections are not circles and the internal hole may degenerate into a segment (Hill's spherical vortex). These rings exhibit exceptional stability properties (see [1,2]), even in presence of viscosity. An incomplete list of papers dealing with fluid vortices and related phenomena is [3-6].

Ring formations of electromagnetic type may appear, intentionally or spontaneously, inside appropriate containers, like in the case of plasma as a consequence of the movement of charges. In other applications, pure electromagnetic waves may find constrained states of equilibrium, where light "rotates" similarly to a non-viscous fluid. These waves enjoy additional features with respect to material fluids, being subject to electrical and magnetic properties. The situation can be ideally replicated by wrapping a wire around a toroid surface and supply it with an alternating current (like in a toroidal transformer). In order to simulate periodic situations, the wavelength of the pulsating current must be a sub-multiple of the length of the perimeter's section. However, periodicity cannot be obtained for all the geometries. For the reasons we are going to detail, the determination of the ring section depends on the behavior of the spectrum of a suitable elliptic operator. In particular, the shape and the magnitude of this region must allow for the existence of at least one eigenvalue with multiplicity greater or equal to two. This paper reviews the main results, with the aim of offering a mathematical background in view of possible practical implementations.

Rotating light is present in a multitude of phenomena. Whispering gallery resonators exist in nature or are built on purpose to force electromagnetic waves to remain trapped (so possibly 
"memorized") into cavities. These waves circulate inside suitable geometrical environments. They are guided through a continuous reflection to turn around, and they may return at the origin with no change of phase, thus forming periodic patterns in time and space. In particular, photonic crystals are complex molecular configurations that may host trapped electromagnetic signals in their reticuli. Optical confinement in microcavities is examined for instance in [7]. There is plenty of papers, either theoretical or practical, on the subject. Here, we only have space to provide a few titles. A direct connection with topological structures associated with rings can be found in [8-12]. Some optical waves trapped in media are studied in [13-15]. Other more conceptual results are given in [16-20].

\section{The Case of the Cylinder}

Our analysis starts from the classical set of Maxwell's equations in vacuum:

$$
\begin{gathered}
\frac{\partial \mathbf{E}}{\partial t}=c^{2} \text { curlB } \\
\operatorname{div} \mathbf{E}=0 \\
\frac{\partial \mathbf{B}}{\partial t}=-\operatorname{curl} \mathbf{E} \\
\operatorname{div} \mathbf{B}=0
\end{gathered}
$$

where $c$ is the speed of light and the two fields $\mathbf{E}$ and $c \mathbf{B}$ have the same dimensions. As usual, Equations (3) and (4) are automatically satisfied, when the electromagnetic fields are expressed through the potentials $\mathbf{A}$ and $\Phi$ such that:

$$
\mathbf{B}=\frac{1}{c} \operatorname{curl} \mathbf{A} \quad \mathbf{E}=-\frac{1}{c} \frac{\partial \mathbf{A}}{\partial t}-\nabla \Phi
$$

We also require that the vector and the scalar potentials are related by the Lorenz gauge condition:

$$
\operatorname{div} \mathbf{A}+\frac{1}{c} \frac{\partial \Phi}{\partial t}=0
$$

Furthermore, one can easily deduce the wave equations:

$$
\begin{aligned}
& \frac{\partial^{2} \mathbf{A}}{\partial t^{2}}-c^{2} \Delta \mathbf{A}=0 \\
& \frac{\partial^{2} \Phi}{\partial t^{2}}-c^{2} \Delta \Phi=0
\end{aligned}
$$

As we are examining periodic solutions, the cylindrical environment can be transformed in a 2-D problem in the system of coordinates $(r, z, \phi)$, where there is no dependence on $z$ (see Figure 1 ). The electric field belongs the plane $(r, \phi)$. The magnetic field is orthogonal and lined up with the $z$-axis. We quickly recall some results concerning electromagnetic waves rotating around the $z$-axis.

Before proceeding, we need to introduce the set of Bessel's functions of the first kind $J_{k}, k \geq 0$, that are implicitly defined through the differential relation:

$$
J_{k}^{\prime \prime}(x)+\frac{J_{k}^{\prime}(x)}{x}-\frac{k^{2} J_{k}(x)}{x^{2}}+J_{k}(x)=0
$$

Other useful properties of Bessel's functions to be recalled are:

$$
J_{k}^{\prime}(x)+\frac{k J_{k}(x)}{x}=J_{k-1}(x)
$$




$$
J_{k+1}(x)=\frac{2 k J_{k}(x)}{x}-J_{k-1}(x)
$$

We remind that $J_{k}(x), k \geq 1$, tends to zero for $x$ tending to zero. Bessel's functions of the second kind are denoted by $Y_{k}, k \geq 0$. They solve the same set of equations, but are unbounded in the neighborhood of $x=0$. A classical reference for this set of special functions is for instance [21].

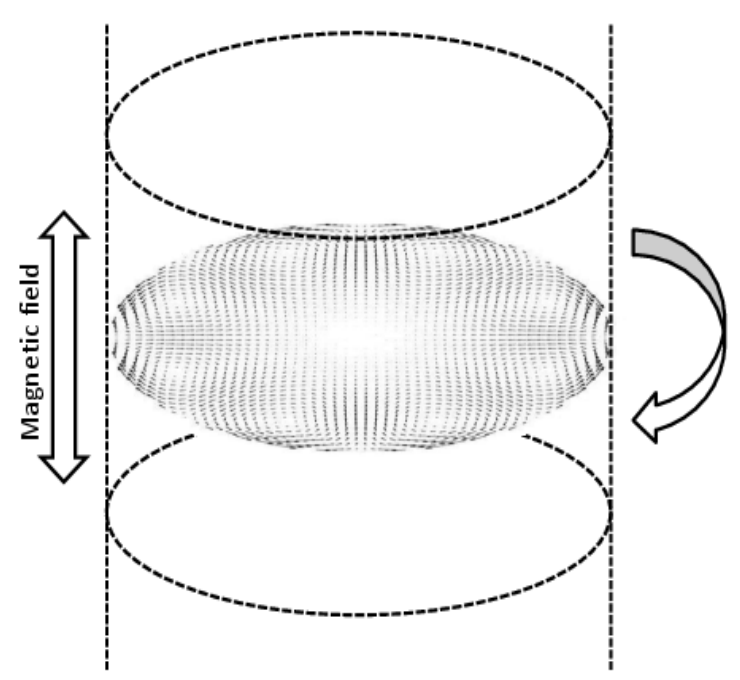

Figure 1. Electric field displacement for a wave circulating in a cylinder as indicated by the arrow. The magnetic field oscillates in the (vertical) direction of the $z$-axis.

By denoting by $\omega>0$ a parameter associated with the frequency of rotation of the wave $\nu=c \omega / \pi$, it turns out that, for certain functions $f$ of the variable $r$, solutions of the Maxwell's equations in vacuum are obtained as:

$$
\begin{gathered}
\mathbf{E}=\left(E_{1}, E_{2}, E_{3}\right)=\left(\frac{k f(\omega r)}{\omega r} \cos (c \omega t-k \phi), 0, f^{\prime}(\omega r) \sin (c \omega t-k \phi)\right) \\
\mathbf{B}=\left(B_{1}, B_{2}, B_{3}\right)=\frac{1}{c}(0, f(\omega r) \cos (c \omega t-k \phi), 0)
\end{gathered}
$$

for any $0 \leq \phi<2 \pi$ and $r \geq 0$. Here, the prime denotes the derivative with respect to $r$. As remarked above, there is no dependency on the variable $z$. These solutions have been proposed in [22], however we do not exclude that they were present in the literature in earlier papers. As a first example, we set $f=J_{k}, k \geq 1$. The corresponding solutions can be checked by direct substitution into Maxwell's equations (it is necessary to use (9)), through a tedious but straightforward calculus exercise. They derive from potentials satisfying the Lorenz's condition (6), i.e.,

$$
\begin{gathered}
\mathbf{A}=\left(A_{1}, A_{2}, A_{3}\right)=-\frac{1}{\omega}\left(J_{k+1}(\omega r) \sin (c \omega t-k \phi), 0, J_{k+1}(\omega r) \cos (c \omega t-k \phi)\right) \\
\Phi=-\frac{1}{\omega} J_{k}(\omega r) \cos (c \omega t-k \phi)
\end{gathered}
$$

where (10) and (11) need to be used.

We observe that $A_{1}$ and $A_{3}$ have a phase difference of $\pi / 2 k$. We can now give a bound to $r$, obtaining in this way field distributions defined on a disk. This cut can be either done by imposing that $f$ is zero at the boundary (Dirichlet type conditions) or by asking that $f^{\prime}$ is zero (Neumann type conditions). The first option states that the boundary is a perfect magnetic conductor (i.e., B has not tangential component on the boundary surface). The last option amounts instead to require that the 
boundary is a perfect conductor ( $E$ lined up with the normal vector). For both cases, the displacement of $\mathbf{E}$ in (12) is shown in Figure 2 for $k=4,6,8$. During time evolution, the vector patterns rigidly rotate around the origin. Fields are defined on disks whose size is inversely proportional to the angular speed of rotation. Since $J_{k}(r)$ behaves as $r^{k}$ near the origin, the intensity of the fields gets smaller at the center of the disk as $k$ increases.
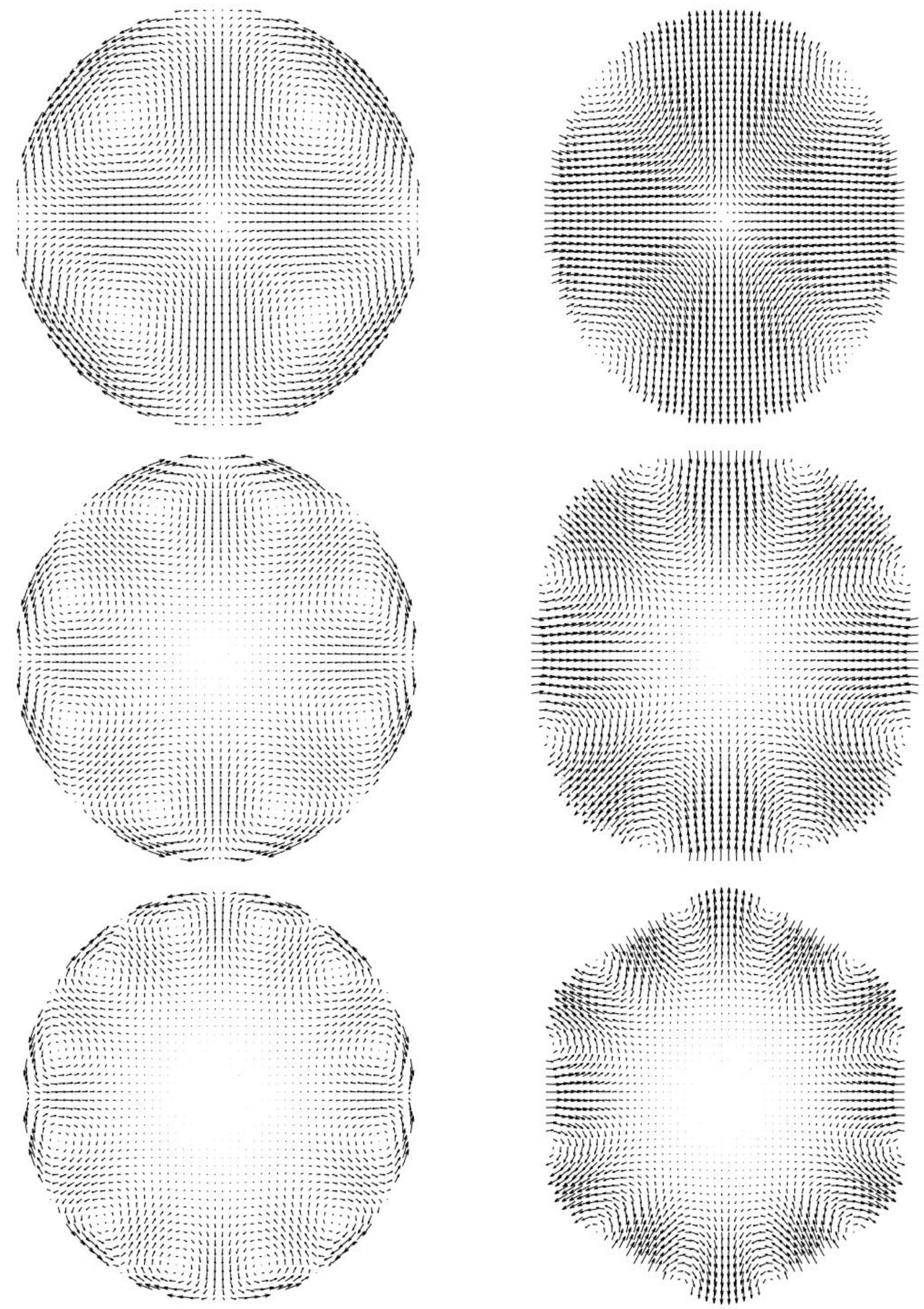

Figure 2. Electric field displacement in a disk as prescribed by (12) for $f=J_{k}$ and $k=2,4,6$. Boundary conditions may be of Dirichlet type (left) or Neumann type (right). The shapes on the right-hand side do not seem to be defined on circles, however this is just an optical illusion. 
Both perfect magnetic and electric conductors are not existing in real life applications. Thus, these concepts represent an ideal abstraction. By the way, the situations here considered seem to be the only ones in which it is possible to come out with analytical solutions. Imperfect electric conductors (see, e.g., [23]) can be treated numerically. A rigorous general approach is however difficult to formalize, since it is not rare to find incongruence between the dynamical Equations (1) and (3), the divergence-free conditions (2) and (4), and certain type of boundary constraints (see also the comments at the beginning of Section 3).

We now observe that $\mathbf{A}$ comes from an eigenvalue problem which is the same as the one we would obtain from the Laplacian, after separation of variables in polar coordinates (see [22] (Section 5.3)). For the sake of simplicity, we use the notation $a(r)=J_{k+1}(\omega r)$. First of all, we can get rid of the dependence on $t$ in the wave Equation (7). Assuming periodicity with respect to $\phi$, the study of the $k$-mode provides the following differential problem for the radial variable:

$$
-\left(\frac{d^{2}}{d r^{2}}+\frac{1}{r} \frac{d}{d r}-\frac{(k+1)^{2}}{r^{2}}\right) a=\omega^{2} a
$$

By introducing the function $w=a^{\prime}+(k+1) a / r$, we have $w=J_{k}$ thanks to (10). To obtain $w$, we have to solve the eigenvalue problem:

$$
L w=-\left(\frac{d^{2}}{d r^{2}}+\frac{1}{r} \frac{d}{d r}-\frac{k^{2}}{r^{2}}\right) w=\lambda w
$$

where $L$ is a positive-definite differential operator. The value of $\lambda>0$ depends on the type of boundary constraints, that are supposed to be imposed at the points $r=0$ and $r=1$. We denote by $\delta_{k, n}$ the $n$-th zero of the Bessel's function $J_{k}$. In the case of homogeneous Dirichlet conditions on the disk $\Omega$ of the plane $(r, \phi)$ having radius equal to 1 , the smallest eigenvalue in (15) is:

$$
\lambda=\delta_{1,1}^{2} \approx 14.6819
$$

where $\delta_{1,1} \approx 3.8317$. This corresponds to the choice $w(r)=J_{1}\left(\delta_{1,1} r\right)$ in (15). Going back to the variables $r$ and $\phi$, the value of $\lambda$ has to be considered with double multiplicity, since the same equation in (15) originates both from the sinus and the cosinus modes. The 2-D related eigenfunctions are orthogonal in $L^{2}(\Omega)$ and show a phase difference of $\pi / 2$. They correspond to the second and the third members of the spectrum of the Laplacian on a disk of radius 1 . Note that the first eigenvalue (not covered by (15)) is always of multiplicity one and there are no eigenvalues with multiplicity greater than two. We also note that $\Phi=0, E_{1}=0, B_{2}=0$ at the boundary of $\Omega$, since $J_{1}\left(\delta_{1,1}\right)=w(1)=0$.

As a general rule, once $\lambda$ has been identified, one can build solutions of the wave equation of the form:

$$
W_{1} \sin (c \sqrt{\lambda} t)+W_{2} \cos (c \sqrt{\lambda} t)
$$

where $W_{1}(r, \phi)=w(r) \cos (k \phi)$ and $W_{2}(r, \phi)=w(r) \sin (k \phi)$ are independent (orthogonal) stationary eigenfunctions. Clearly the above expression solves the scalar wave equation in vacuum. In fact, the second derivative in time produces the multiplicative factor $-c^{2} \lambda$, while the application of the Laplace operator in cylindrical coordinates is equivalent to a multiplication by $-\lambda$.

Extensions of the previous results may be obtained when the rotation around the axis is combined with a radial oscillation. Here, in order to find exact solutions, we must assume that the section has a central hole. The study in [24] is connected to the search of eigenfunctions for the Laplace operator in such a way that the dimension of the corresponding eigenspace is equal to four. In such a circumstance, $f$ is a linear combination of $J_{k}$ and $Y_{k}$. Note that we have to stay away from the point $r=0$, since Bessel's functions of the second kind are singular at the origin. Again, the magnetic field is parallel to the cylinder's axis and the electric field belongs to the plane $(r, \phi)$. One may consider sections where $R_{m}=1 \leq r \leq R_{M}$ with the boundary conditions imposed at $r=R_{m}$ and $r=R_{M}$. 
These last can be either of Dirichlet or Neumann type. Geometries compatible with the existence of solutions can be obtained by adjusting $r=R_{m}$ and $r=R_{M}$, as suggested by the location of the zeros of suitable combinations of Bessel's functions. Several options are possible and some of them are documented in [24].

Once the frequency of rotation (depending on $\omega$ ) is fixed and the type of boundary conditions decided, the size of the domain is automatically determined. The magnitude scales inversely with the constant $\omega$ (high frequencies, small objects, and vice versa). This is done by preserving the geometrical proportions, thanks to the linearity of Maxwell's equations. There is however a reduced number of domains allowing for the creation of these periodic waves. If the proper shape is not respected, the wave does not follow a time-periodic motion, i.e., it will not complete an entire cycle preserving the phase. As said above, a sufficient condition allowing for such a guided behavior is related to the multiplicity of the eigenvalues of the Laplace operator on the specific domain.

Concerning the solutions proposed so far, there are some peculiarities to be pointed out. First of all, the information travels along concentric circumferences at constant angular velocity and there is not a proper wave-front that envelops the lines of force of the electric field. The speed of rotation varies from zero at the center with no upper limit when moving far away. Assuming zero boundary conditions for the magnetic field, the smallest domain containing the wave has a radius where the peripheral velocity exceeds that of light. Contrary to what is commonly believed, Maxwell's equations are perfectly compatible with this type of behavior. Such a paradox can be solved in the context of general relativity, where we may suppose that a suitable alteration of the space-time ensures the development at constant velocity $c$ along curved geodesics. The problem is however not trivial and requires further analysis (not available at the moment). The second comment concerns with the fact that $\mathbf{E}$ is orthogonal to $\mathbf{B}$, but not to the propagation fronts. Indeed, during the evolution, $\mathbf{E}$ has both transverse and longitudinal oscillating components. This is also in contrast with popular belief. Locally, this behavior is similar to the one displayed by a wave traveling in a medium with dielectric constant different from that of vacuum, which is not however the case we are dealing with in this section. On the other hand, curly waves are the primary ingredients that inspired Maxwell to develop his theory (see [25]). The aim was to investigate the electromagnetic structure of matter in terms of everlasting circulant "molecular vortices". Though the original Maxwell's equations have been lately modified by other authors to become those expressed by (1)-(4), the particular rotating solutions remain to testify for his construction. Similar considerations are the fulcrum that led in [26] to search for a sort of a classical explanations of quantum phenomena.

The evolution in a thin toroid can be approximated, with a rather good level of accuracy, starting from the cylinder's versions. For more general geometries, the study will be faced in the coming section.

\section{The Case of the Ring}

The classical equations of electromagnetism in vacuum are well-suited for solutions confined in steady ring-shaped domains. This is made possible by the orthogonality of $\mathbf{E}$ and $\mathbf{B}$, as well as by the enforcement of the two divergence-free conditions ( $\operatorname{div} \mathbf{E}=0$ and $\operatorname{div} \mathbf{B}=0)$. Indeed, when the the two sets of lines of force remain closed and orthogonal, the toroid topology comes very natural. These are typical circumstances where all the eight Equations (1)-(4) (two of scalar type and two of vector type) in the six unknowns $E_{1}, E_{2}, E_{3}, B_{1}, B_{2}, B_{3}$ can be totally fulfilled. Other (classical) instances only lead to a partial resolution of the set of Maxwell's equations in vacuum. As pointed out in [27-29], there is plenty of examples where at least one of the equations may remain unsatisfied. This is mainly due to the impossibility to enforce at the same time the wave equation, the divergence-free relation and the boundary conditions.

As in the previous section, the recipe is the study of the lower spectrum of a suitable elliptic operator, depending on the shape and magnitude of the sections. In cylindrical coordinates $(r, z, \phi)$, we introduce the potentials: 


$$
\mathbf{A}=\frac{1}{c}(a(t, r, z), b(t, r, z), 0) \quad \Phi=-\left(\frac{\partial A}{\partial r}+\frac{A}{r}+\frac{\partial B}{\partial z}\right)
$$

where $a$ and $b$ are the unknowns to be computed, whereas $A$ and $B$ are their primitives with respect to the time variable. The Lorenz condition (6) turns out to be automatically satisfied. The functions $a$ and $b$ are defined in a region $\Omega$ of the plane $(r, z)$. Differently from the previous case, $\Omega$ is vertically oriented. Since there is no dependence on $\phi$, the solutions can be naturally extended to a toroid $\Sigma$ with section $\Omega$. The axis of $\Sigma$ will be parallel to the $z$-axis (see Figure 3).

From (18) we deduce the electromagnetic fields:

$$
\mathbf{E}=\left(-\frac{\partial \Phi}{\partial r}-\frac{1}{c^{2}} \frac{\partial a}{\partial t},-\frac{\partial \Phi}{\partial z}-\frac{1}{c^{2}} \frac{\partial b}{\partial t}, 0\right) \quad \mathbf{B}=\frac{1}{c^{2}}\left(0,0, \frac{\partial b}{\partial r}-\frac{\partial a}{\partial z}\right)
$$

By requiring that $\mathbf{E}$ and $\mathbf{B}$ satisfy (1), we arrive at the following system:

$$
\begin{gathered}
\frac{1}{c^{2}} \frac{\partial^{2} a}{\partial t^{2}}=\frac{\partial^{2} a}{\partial z^{2}}+\frac{\partial}{\partial r}\left(\frac{\partial a}{\partial r}+\frac{a}{r}\right) \\
\frac{1}{c^{2}} \frac{\partial^{2} b}{\partial t^{2}}=\frac{\partial^{2} b}{\partial z^{2}}+\frac{\partial^{2} b}{\partial r^{2}}+\frac{1}{r} \frac{\partial b}{\partial r}
\end{gathered}
$$

A possible way to couple $a$ and $b$ is through the boundary condition on $\partial \Omega$ :

$$
\frac{\partial a}{\partial z}=\frac{\partial b}{\partial r}
$$

though other options can be examined. Relation (22) amounts to require that $\mathbf{B}=c^{-1} \operatorname{curl} \mathbf{A}=0$ at the contour of $\Omega$. In this way the field $\mathbf{E}$ is tangential to the boundary. Indeed, thanks to (18), (20) and (21), one can write:

$$
\mathbf{E}=\left(-\frac{\partial}{\partial z}\left(\frac{\partial A}{\partial z}-\frac{\partial B}{\partial r}\right), \frac{1}{r} \frac{\partial}{\partial r}\left(r \frac{\partial A}{\partial z}-r \frac{\partial B}{\partial r}\right), 0\right)
$$

which implies that $\mathbf{E}$ is orthogonal to the gradient of the potential $r(\partial A / \partial z-\partial B / \partial r)$. Since (22) is valid for all $t$, such a potential vanishes at the boundary of $\Omega$, so that its gradient is orthogonal to $\partial \Omega$. As a consequence $\mathbf{E}$ is tangential. Other conditions, such as perfect conductivity ( $\mathbf{E}$ orthogonal to the boundary), can be easily implemented. In this case, we need to set $E_{2}=0$ in (23), so that condition (22) becomes:

$$
\frac{\partial(r a)}{\partial z}=\frac{\partial(r b)}{\partial r}
$$

The domain $\Omega$ (which is not necessarily a circle) is supposed to be "centered" at the point $(\eta, 0)$, where $\eta>0$ is large enough to avoid the intersection of $\Omega$ with the $z$-axis. Thus, the quantity $2 \eta$ is related to the major diameter of the toroid. As done in [22] (Section 5.4), we set $y=r-\eta$. By differentiating (20) with respect to $z$ and (21) with respect to $y$, we arrive at:

$$
\left\{\begin{array}{l}
\frac{1}{c^{2}} \frac{\partial^{2} u}{\partial t^{2}}=\frac{\partial^{2} u}{\partial z^{2}}+\frac{\partial}{\partial y}\left(\frac{\partial u}{\partial y}+\frac{u}{y+\eta}\right) \\
\frac{1}{c^{2}} \frac{\partial^{2} v}{\partial t^{2}}=\frac{\partial^{2} v}{\partial z^{2}}+\frac{\partial}{\partial y}\left(\frac{\partial v}{\partial y}+\frac{v}{y+\eta}\right)
\end{array}\right.
$$

where $u=\partial a / \partial z$ and $v=\partial b / \partial r=\partial b / \partial y$, with $u-v=0$ on $\partial \Omega$. Through the substitutions $\tilde{u}=u \sqrt{y+\eta}, \tilde{v}=v \sqrt{y+\eta}$, we then obtain: 


$$
\left\{\begin{array}{l}
\frac{1}{c^{2}} \frac{\partial^{2} \tilde{u}}{\partial t^{2}}=\frac{\partial^{2} \tilde{u}}{\partial z^{2}}+\frac{\partial^{2} \tilde{u}}{\partial y^{2}}-\frac{3}{4} \frac{\tilde{u}}{(y+\eta)^{2}} \\
\frac{1}{c^{2}} \frac{\partial^{2} \tilde{v}}{\partial t^{2}}=\frac{\partial^{2} \tilde{v}}{\partial z^{2}}+\frac{\partial^{2} \tilde{v}}{\partial y^{2}}-\frac{3}{4} \frac{\tilde{v}}{(y+\eta)^{2}}
\end{array}\right.
$$

to be solved in $\Omega$, with the boundary condition: $\tilde{u}-\tilde{v}=0$.

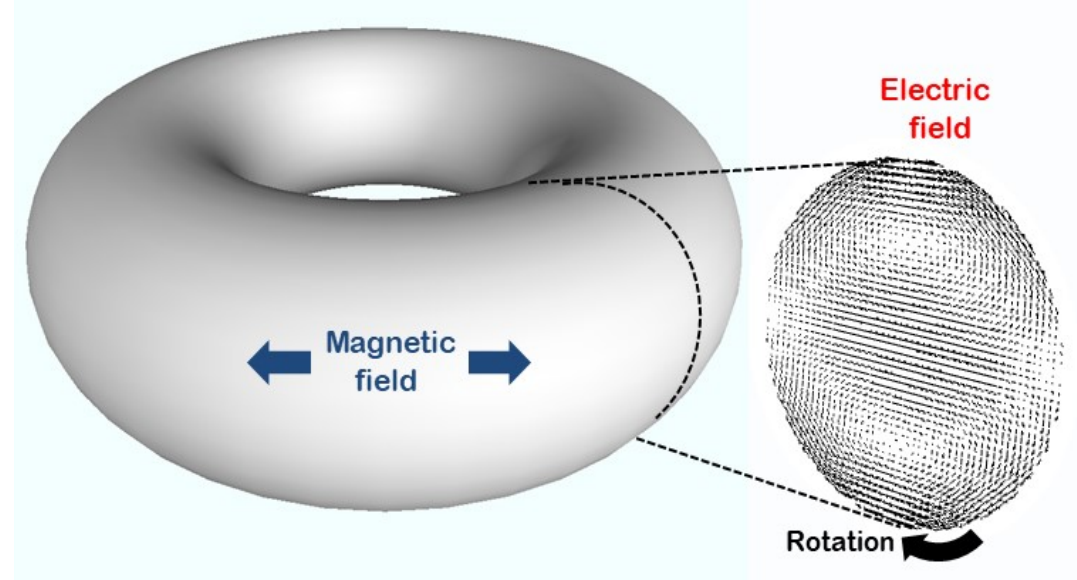

Figure 3. Electromagnetic fields circulating in a toroid cavity $\Sigma$. The electric field lies on each vertical section, whereas the magnetic one is distributed parallel to the large circumference. The time evolution recalls that of a fluid dynamics vortex ring.

Furthermore, we introduce a new unknown $w=\tilde{u}-\tilde{v}$ and take the difference of the two equations in (26). Finally, we pass to the stationary eigenvalue problem:

$$
\begin{cases}L w=-\frac{\partial^{2} w}{\partial z^{2}}-\frac{\partial^{2} w}{\partial y^{2}}+\frac{3}{4} \frac{w}{(y+\eta)^{2}}=\lambda w \text { in } \Omega \\ w=0 \quad \text { on } \partial \Omega\end{cases}
$$

obtained by looking for time-periodic solutions of the system in (26).

Similarly to the previous section, we would like $\lambda>0$ to be an eigenvalue (with multiplicity two, at least) of the positive-definite differential operator: $L=-\partial^{2} / \partial z^{2}-\partial^{2} / \partial y^{2}+\frac{3}{4}(y+\eta)^{-2}$, with homogeneous Dirichlet boundary conditions. This request implicitly defines the set $\Omega$. By introducing:

$$
\begin{gathered}
{[u(t, y, z)-v(t, y, z)] \sqrt{y+\eta}=\tilde{u}(t, y, z)-\tilde{v}(t, y, z)} \\
=w_{1}(y, z) \sin (c t \sqrt{\lambda})+w_{2}(y, z) \cos (c t \sqrt{\lambda})
\end{gathered}
$$

where $w_{1}$ and $w_{2}$ are independent (suitably normalized) eigenfunctions corresponding to $\lambda$, one gets viable solutions of the system (26). The electromagnetic fields are then recovered by the expressions (see (23) and (19)):

$$
\begin{gathered}
\mathbf{E}=\frac{1}{c \sqrt{\lambda}}\left(-\frac{\partial}{\partial z}\left(\frac{-w_{1} \cos \zeta+w_{2} \sin \zeta}{\sqrt{y+\eta}}\right), \frac{1}{y+\eta} \frac{\partial}{\partial y}\left(\left(-w_{1} \cos \zeta+w_{2} \sin \zeta\right) \sqrt{y+\eta}\right), 0\right) \\
\mathbf{B}=\frac{1}{c^{2}}\left(0,0, \frac{w_{1} \sin \zeta+w_{2} \cos \zeta}{\sqrt{y+\eta}}\right)
\end{gathered}
$$


with $\zeta=c t \sqrt{\lambda}$. The above displacements rotate in the $(r, z)$ plane by varying the time parameter $t$.

At this point, we are able to define the 3-D set $\Sigma=\Omega \times[0,2 \pi[$ (see Figure 3), where the corresponding solution does not depend on $\phi$. As mentioned in the introduction, in fluid dynamics this structure is known as a vortex ring (see for instance [30] (Section 7.2)).

In the electrodynamic case considered above, on the surface of $\Sigma$, the field $\mathbf{E}$ is tangential whereas B is zero. By the way, as mentioned before, other types of boundary conditions may be taken into account. To know more about the shape of $\Sigma$, we refer to [31], where a series of numerical experiments have been performed with the aim of determining suitable sets $\Omega$ for which the fourth and the fifth eigenvalues of (27) are coincident, obtaining very peculiar rotating configurations, among infinite many possible situations. The angular velocity of rotation is proportional to $\sqrt{\lambda}$. The most simple dynamics are those corresponding to eigenvalues belonging to the lower part of the spectrum (i.e., related to low frequency modes), and with $\Omega$ convex and more rounded as possible. In [31], spherical vortex rings of Hill's type (where the hole is reduced to a segment) have been also analyzed numerically. These results have an impressive similarity with the more recent ones discussed in [32], obtained in the framework of plasma physics.

All the above situations are however rather critical. With this we mean that little modifications of the shape of $\Sigma$ bring to non perfectly periodic solutions. This is also true if we modify the boundary conditions (see the comments given in Section 2). The dynamics in these cases depends on several harmonics, thus the entire spectrum of the differential operator $L$ is going to be involved. Note that when $\eta$ is suitably large with respect to the size of $\Omega$, we are practically returning to the case of the cylinder studied in the previous section, where exact solutions are available when $\Omega$ is a disk.

\section{Extensions and Applications}

By varying the constant $c$, it is possible to handle solutions defined inside a homogeneous dielectric, where the electromagnetic message revolves at velocities lower than that of light. More theoretically difficult, but numerically feasible, is the situation where the dielectric is not homogeneous.

This research on electromagnetic rings was inspired by the possibility of building stable elementary particles, such as the electron (see $[22,26]$ and the references therein). The idea is not new and, starting from the original paper [33], many authors have devolved their efforts to its realization. The results in [26] rely on an extension of the electrodynamics equations that allow to handle regions where the divergence of $\mathbf{E}$ is different from zero even in vacuum. With this trick it is possible to add to the ring solution other properties, able to confer a net charge and a mass to the particle model. By following such an approach it is also possible to enlarge the solution space of Maxwell's equation by including solitary waves with compact support (solitons). Such an improvement is certainly of interest for the understanding of photons and optical phenomena in general (see [34]). It is not our aim here to further discuss the merits of this approach, however we believe that it may be of high interest in the study of both theoretical issues and innovative practical applications.

A simple experiment puts in relation magnetic fields with topological properties (see [35]). Let us suppose that two simply-connected pieces of non magnetized iron, when joined together, form an annular shape. A conductive wire inserted into the hole is supplied for a fraction of time with an electric current. This generates closed magnetic lines of force inside the iron. After the removal of the wire, the two irons are solidly glued together. Such an adhesive contact may last for long time after the current shut off. When torn apart, the two components do not reconnect anymore. Thus, the breakage of the toroid passes through a topological modification that also changes its magnetic status. The energy that was stored within the ring comes out in some form after the rupture. This observation somehow confirms how important is to work with doubly-connected geometries and organize the experiments in such a way the magnetic field is distributed along closed patterns embedded in the ring support.

The study here developed in vacuum, reveals original configurations and may help in the analysis of confined plasma (see, e.g., [36,37]), with special attention to the detection of quantum vortex rings in superfluid helium (see [38] for an overview). Further applications are found in the field 
of whispering gallery resonators. Waves trapped in these cavities are smoothly guided to circulate around by continuous reflection returning at the origin with the initial phase. Spherical, cylindrical or ring-shaped whispering galleries are produced for a broad range of industrial applications, for instance in fiber telecommunications technologies or biosensing. Solitary waves in rings are commonly produced in optical laboratories by sending laser beams of appropriate frequency through a crystal lattice. A few non specialized publications are [39,40]. Some references on more specific applications are found in the introductory section of this paper.

As far as we know, the insertion or the emission of the signal in whispering galleries (annuli or disks) follows in general a path tangential to the major circumference. Indicative references are [41-44], from the experimental viewpoint, and $[45,46]$, concerning a more theoretical approach. From the results of this paper, it turns out that this procedure would not be optimal, in order to ensure a long-time storage on the information packets. Instead, we believe that photons (of higher frequency) should reach the device from directions tangential to the minor circumference, as depicted in Figure 4. This is also true by virtue of the property stated above in this section, that the closed lines of force of the magnetic field must be strictly contained within the dielectric body, with the aim to reinforce the stability of the stored signal. In any case, we do not have any idea how to technically implement such a result.

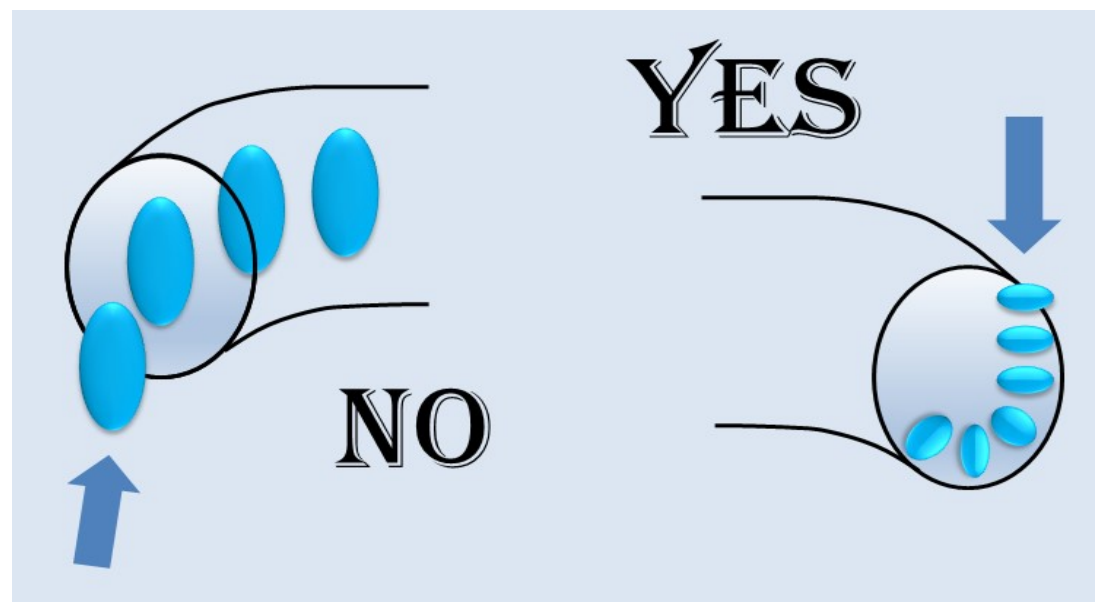

Figure 4. Different ways to send wave-packets in a toroid whispering gallery. According to what suggested in this paper, the signal should not approach the device by staying parallel to the major diameter (left), but rather following the orthogonal direction (right).

Finally, in the atmospheric phenomenon known as ball lightning (see, e.g., $[47,48]$ ), some form of radiation stays constrained for minutes in confined erratic regions. A hypothesis is that such objects also originate from electromagnetic waves trapped in bubbles $[49,50]$, or more elaborated shapes [51-54], including rings.

\section{Conclusions}

We think that the results here collected may have a general validity independently on the field of application. Indeed, the study of the determination of periodic solutions from the analysis of the spectrum of suitable differential operators, may be applied in several circumstances. The search for electromagnetic waves trapped in a toroid suggests interesting mathematical questions. The behavior is similar to that of non-viscous fluids confined in ring vortices, together with additional magnetic properties. The dynamics critically depend on the shape of the support and the boundary conditions imposed. Numerical simulations are then necessary to sort out reliable configurations.

Our analysis might be the starting point for new applications, that at the moment we are unable to predict. We are not enough experienced to come out with practical suggestions. This paper may however be of inspiration to the interested reader for creating new tools or improving the existing ones. 
Funding: This research received no external funding.

Conflicts of Interest: The author declares no conflict of interest.

\section{References}

1. Maxworthy, T.J. The structure and stability of vortex rings. J. Fluid Mech. 1972, 51, 15-32. [CrossRef]

2. Lim, T.T.; Nickels, T.B. Vortex rings. In Fluid Vortices; Green, S.I., Ed.; Kluwer Academic: Dordrecht, The Netherlands, 1995.

3. Pullin, D.I. Vortex ring formation at tube and orifice openings. Phys. Fluids 1979, 22, 401-403. [CrossRef]

4. Shariff, K.; Leonard, A. Vortex rings. Annu. Rev. Fluid Mech. 1992, 24, 235-279. [CrossRef]

5. Sullivan, I.S.; Niemela, J.J.; Hershberger, R.E.; Bolster, D.; Donnelly, R.J. Dynamics of thin vortex rings. J. Fluid Mech. 2008, 609, 319-347. [CrossRef]

6. Wakelin, S.L.; Riley, N. On the formation of vortex rings and pairs of vortex rings. J. Fluid Mech. 1997, 332, 121-139. [CrossRef]

7. Benson, T.M.; Boriskina, S.V.; Sewell, P.; Vukovic, A.; Greedy, S.C.; Nosich, A.I. Micro-optical resonators for microlasers and integrated optoelectronics: Recent advances and future challenges. In Frontiers of Planar Lightwave Circuit Technology, Simulation and Fabrication; Janz, S., Ctyroky, J., Tanev, S., Eds.; Springer: Berlin/Heidelberg, Germany, 2005; pp. 39-70.

8. Kevrekidis, P.G.; Nistazakis, H.E.; Frantzeskakis, D.J.; Malomed, B.A.; Bishop, A.R. Ring solitons on vortices. Phys. Rev. E 2001, 64, 066611. [CrossRef]

9. Bigelow, M.S.; Zerom, P.; Boyd, R.W. Breakup of ring beams carrying orbital angular momentum in sodium vapor. Phys. Rev. Lett. 2004, 92, 083902. [CrossRef]

10. Efremidis, N.K.; Hizanidis, K.; Malomed, B.A.; Di Trapani, P. Three dimensional vortex solitons in self-defocusing media. Phys. Rev. Lett. 2007, 98, 113901. [CrossRef]

11. Fischer, A.M.; Campo, V.L., Jr.; Portnoi, M.E.; Römer, R.A. Exciton storage in a nano-scale Aharonov-Bohm ring with electric field tuning. Phys. Rev. Lett. 2009, 102, 096405. [CrossRef]

12. Papasimakis, N.; Fedotov, V.A.; Savinov, V.; Raybould, T.A.; Zheludev, N.I. Electromagnetic toroidal excitations in matter and free space. Nat. Mater. 2016, 15, 263-271. [CrossRef]

13. Kruglov, V.I.; Vlasov, R.A. Spiral self-trapping propagation of optical beams in media with cubic nonlinearity. Phys. Lett. A 1985, 111, 401-404. [CrossRef]

14. Boriskina, S.V. Coupling of whispering-gallery modes in size-mismatched microdisk photonic molecules. Opt. Lett. 2007, 32, 1557-1559. [CrossRef] [PubMed]

15. Shen, J.-T.; Fan, S. Strongly correlated two-photon transport in a one-dimensional waveguide coupled to a two-level system. Phys. Rev. Lett. 2007, 98, 153003. [CrossRef] [PubMed]

16. Mabuchi, H.; Kimble, H.J. Atom galleries for whispering atoms: Binding atoms in stable orbits around an optical resonator. Opt. Lett. 1993, 19, 749-751. [CrossRef]

17. Firth, W.J.; Skryabin, D.V. Optical solitons carrying orbital angular momentum. Phys. Rev. Lett. 1997, 79, 2450-2453. [CrossRef]

18. Karzig, T.; Bardyn, C.-E.; Lindner, N.H.; Refael, G. Topological polaritons. Phys. Rev. X 2015, 5, 031001. [CrossRef]

19. He, Y.J.; Malomed, B.A.; Wang, H.Z. Steering the motion of rotary solitons in radial lattices. Phys. Rev. A 2007, 76, 053601. [CrossRef]

20. Kamor, A.; Mauger, F.; Chandre, C.; Uzer, T. Annular billiard dynamics in a circularly polarized strong laser field. Phys. Rev. E 2012, 85, 016204. [CrossRef]

21. Watson, G.N. A Treatise on the Theory of Bessel Functions, 2nd ed.; Cambridge University Press: Cambridge, UK, 1995.

22. Funaro, D. Electromagnetism and the Structure of Matter; World Scientific: Singapore, 2008.

23. Hurley, D.G. Boundary conditions for thin imperfect conductors and insulators. Geophys. Perspect. 1975, 25, 70-79. [CrossRef]

24. Funaro, D. Trapping electromagnetic solitons in cylinders. Math. Model. Anal. 2014, 19, 44-51. [CrossRef]

25. Maxwell, J.C. On physical lines of forces. Lond. Edinb. Dubl. Phil. Mag. 1861, 161-175, 281-291. [CrossRef]

26. Funaro, D. From Photons to Atoms: The Electromagnetic Nature of Matter; World Scientific: Singapore, 2020.

27. Engelhardt, W. On the Solvability of Maxwell's Equations. Ann. Fond. Louis de Broglie 2012, 37, 3-14. 
28. Funaro, D. Charging capacitors according to Maxwell's equations: Impossible. Ann. Fond. Louis de Broglie 2014, 39, 1-16.

29. Funaro, D. High frequency electrical oscillations in cavities. Math. Model. Anal. 2018, 23, 345-358. [CrossRef]

30. Batchelor, G.K. An Introduction to Fluid Dynamics; Cambridge University Press: Cambridge, UK, 1967.

31. Chinosi, C.; Della Croce, L.; Funaro, D. Rotating electromagnetic waves in toroid-shaped regions. Int. J. Mod. Phys. C 2010, 21, 11-32. [CrossRef]

32. Hsueh, C.-H.; Gou, S.-C.; Horng, T.-L.; Kao, Y.-M. Vortex-ring solutions of the Gross-Pitaevskii equation for an axisymmetrically trapped Bose-Einstein condensate. J. Phys. B 2007, 40, 4561-4571. [CrossRef]

33. Poincaré, M.H. Sur la dynamique de l'électron. Rend. Circ. Palermo 1960, XXI, 129-175. [CrossRef]

34. Funaro, D. Numerical simulation of electromagnetic solitons and their interaction with matter. J. Sci. Comput. 2010, 45, 259-271. [CrossRef]

35. Leedskalnin, E. Magnetic Current; Personal Copyright: Homestead, FL, USA, 1945.

36. Chen, C.; Pakter, R.; Seward, D.C. Equilibrium and stability properties of self-organized spiral toroids. Phys. Plasmas 2001, 8, 4441-4449. [CrossRef]

37. Hazeltine, R.D.; Meiss, J.D. Plasma Confinement; Dover: Mineola, NY, USA, 2003.

38. Barenghi, C.F.; Donnelly, R.J. Vortex rings in classical and quantum systems. Fluid Dyn. Res. 2009, 41, 051401. [CrossRef]

39. Oraevsky, A.N. Whispering-gallery waves. Quantum Electron. 2002, 32, 377-400. [CrossRef]

40. Snyder, A.; Love, J. Optical Waveguide Theory; Kluwer Academic: Dordrecht, The Netherlands, 1983.

41. Al-Attili, A.Z.; Kako, S.; Husain, M.K.; Gardes, F.Y.; Higashitarumizu, N.; Iwamoto, S.; Arakawa, Y.; Ishikawa, Y.; Arimoto, H.; Oda, K.; et al. Whispering gallery mode resonances from Ge micro-disks on suspended beams. Front. Mater. 2015, 2, 43-58. [CrossRef]

42. Lu, X.; Li, Q.; Westly, D.A.; Moille, G.; Singh, A.; Anant, V.; Srinivasan, K. Chip-integrated visible-telecom entangled photon pair source for quantum communication. Nat. Phys. 2019, 15, 373-381. [CrossRef] [PubMed]

43. Kwon, O.; Sheen, M.H.; Kim, Y.C. Photonic quantum ring laser of whispering cave mode. In Advances in Optical and Photonic Devices; Kim, K.Y., Ed.; IntechOpen: London, UK, 2016.

44. Preu, S.; Schmid, S.I.; Sedlmeir, F.; Evers, J.; Schwefel, H.G.L. Directional emission of dielectric disks with a finite scatterer in the THz regime. Opt. Express 2013, 21, 16370-16380. [CrossRef]

45. Raskhodchikov, A.V.; Raskhodchikov, D.V.; Scherbak, S.A.; Lipovskii, A.A. Numerical simulation of eigenmodes of ring and race-track optical microresonators. IOP J. Phys. Conf. Ser. 2017, 917, 062040. [CrossRef]

46. Xiao, Y.F.; Zou, C.L.; Li, B.B.; Li, Y.; Dong, C.H.; Han, Z.F.; Gong, Q. High-Q exterior whispering-gallery modes in a metal-coated microresonator. Phys. Rev. Lett. 2010, 105, 153902. [CrossRef]

47. Bychkov, V.L.; Golubkov, G.V.; Nikitin, A.I. (Eds.) The Atmosphere and Ionosphere, Elementary Processes, Monitoring and Ball Lightning; Springer: Berlin/Heidelberg, Germany, 2014.

48. Stenhoff, M. Ball Lightning: An Unsolved Problem in Atmospheric Physics; Kluwer Academic: Dordrecht, The Netherlands, 2002.

49. Dawson, G.A.; Jones, R.C. Ball lightning as a radiation bubble. Pure Appl. Geophys. 1969, 75, $247-262$. [CrossRef]

50. Endean, V.G. BL as electromagnetic radiation. Nature 1976, 263, 753-755. [CrossRef]

51. Alanakyan, Y.R. Energy capacity of an electromagnetic vortex in the atmosphere. J. Exp. Theor. Phys. 1994, 78, 320-326.

52. Baraov, A. Electromagnetic induction and relativistic double layer: Mechanism for ball lightning formation. Proceedings 2019, 33, 3. [CrossRef]

53. Funaro, D. A Model for ball lightning derived from an extension of the electrodynamics equations. In Proceedings of the VI International Conference on Atmosphere, Ionosphere, Safety, Kaliningrad, Russia, 3-9 June 2018.

54. Rañada, A.F.; Trueba, J.L. Ball lightning an electromagnetic knot? Nature 1996, 383, 32. [CrossRef]

(C) 2020 by the authors. Licensee MDPI, Basel, Switzerland. This article is an open access article distributed under the terms and conditions of the Creative Commons Attribution (CC BY) license (http://creativecommons.org/licenses/by/4.0/). 